\title{
Experiencias Exitosas con las TIC: Recurso Pedagógico en la Enseñanza de Finanzas Internacionales
}

\author{
Successful Experiences with ICT: Pedagogical Resource in Teaching International \\ Finance
}

German Enrique Cárdenas-Contreras ${ }^{1}$

\section{( EDIRIN. RTED}

Recibido: 18/agosto/2021

Aceptado: 17/noviembre/2021

Publicado: 29/noviembre/2021

Páginas: 174-182

\&aís

${ }^{1}$ Colombia

\section{IIII Institución}

${ }^{1}$ Universidad de Pamplona

\section{Correo Eletrónico}

1'german.cardenas@unipamplona.edu.co

\section{ORCID}

${ }^{1}$ https://orcid.org/0000-0002-1343-935X

\section{Citar así: C APA / IEEE}

Cárdenas-Contreras, G. (2021) Experiencias Exitosas con las TIC: Recurso Pedagógico en la Enseñanza de Finanzas Internacionales. Revista TecnológicaEducativa Docentes 2.0, 1(1), 174-182. https://doi.org/10.37843/rted.v1i1.272

G. Cárdenas-Contreras, "Experiencias Exitosas con las TIC: Recurso Pedagógico en la Enseñanza de Finanzas Internacionales", RTED, vol. $1, \mathrm{n} .^{\circ} 1, \mathrm{pp}$. 174-182, nov. 2021.

\section{Resumen}

En el panorama mundial, las Tecnologías de Información y Comunicación (TIC), forman parte de la política educativa en los países, sobre todo los más avanzados. El presente artículo tuvo como propósito determinar la influencia que han tenido las tecnologías educativas, en los procesos de enseñanza de finanzas internacionales en estudiantes universitarios. El proceso realizado desde una visión pedagógica se fundamentó, desde los cimientos teóricos del conectivismo, el cual explica el aprendizaje complejo en un mundo social digital en rápida evolución. La estructura metodológica se orientó desde un enfoque cualitativo de corte documental, con un tipo descriptivo; para la recolección de información, se utilizó como instrumento, una ficha de registro bibliográfico, en donde mediante un procedimiento de revisión documental, se recopilaron y analizaron seis experiencias exitosas relacionadas al ámbito de las finanzas y ciencias económicas, tanto en el contexto internacional, como a nivel nacional. Los resultados obtenidos, dieron muestra de que las TIC han tenido un impacto significativo, llegando a presentar un crecimiento exponencial en los contextos educativos, situación que llevó a que la dinámica entre docentes y estudiantes estuviera mediada por tecnologías, sin embargo, en el ámbito financiero existe poca cantidad de experiencias exitosas que reflejen el impacto de las tecnologías, hecho que evidencia falencias pedagógicas en los docentes de economía, siendo indispensable fomentar el uso de herramientas TIC y recursos digitales en sus prácticas pedagógicas.

Palabras clave: Conectivismo, experiencias exitosas, finanzas internacionales, TIC.

\section{Abstract}

On the world scene, Information and Communication Technologies (ICT) are part of educational policy in countries, especially the most advanced ones. The purpose of this article was to determine the influence that educational technologies have had on the teaching processes of international finance in university students. The process carried out from a pedagogical perspective was founded from the theoretical foundations of connectivism, which explains complex learning in a rapidly evolving digital social world. In the methodological structure, the study was oriented from a qualitative documentary approach with a descriptive type. For a collection of information, a bibliographic record card was used as an instrument, were using a document review procedure, six successful experiences related to the field of finance and economic sciences were collected and analyzed, both in the international context, as well as at the national level. The results obtained show that ICTs have had a significant impact, reaching exponential growth in educational contexts, since the declaration of a pandemic issued by the WHO. This situation led to the dynamics between teachers and students being mediated by technology; however, in the financial field, few successful experiences reflect the impact of technologies, a fact that shows pedagogical shortcomings in economics teachers, being essential to promote the use of ICT tools and digital resources in their pedagogical practices teaching.

Keywords: Successful experiences, international finance, pedagogical resource, ICT. 


\section{Introducción}

En el panorama mundial, las Tecnologías de Información y Comunicación (TIC), forman parte de la política educativa en los países, sobre todo los más avanzados. Así, por ejemplo, en países como Chile se ha impulsado un componente estratégico de su reforma educacional. En este caso, según lo plantea López (2006) las TIC se insertan bajo dos premisas: el mejoramiento de la equidad, al reducir la brecha de acceso a la información en las escuelas; y el mejoramiento de la calidad educacional. En los últimos años las diferentes tecnologías han incursionado en las actividades cotidianas de las personas, ayudando a resolver problemas, satisfacer necesidades o para que las actividades rutinarias sean menos dispendiosas, es por ello por lo que al hablar de tecnología es fundamental tener claridad sobre lo que concierne a estas.

Resulta fundamental mencionar que, los países latinoamericanos toman como ejemplo los avances de los países del primer mundo. En relación directa con este planteamiento, el Ministerio de Educación Nacional, MEN (2016) menciona la necesidad de generar una visión propia, que atienda la realidad de los países de América Latina, la cual parta de una cultura integradora, donde los factores sociales que inciden en la capacidad innovadora sean correctamente interpretados. "La incursión de las nuevas tecnologías en los contextos cotidianos ha hecho que estén presentes en las diferentes esferas de la sociedad" (Sandoval, 2020, p.25).

Así mismo, Barragán \& Vela refieren que, "los países emergentes deben poner en marcha reformas integrales en cada uno de ellos que les permitan mejorar los tratados de libre comercio internacional y mejorar la competitividad de los mismos y sus regiones" (2015, p. 30). Por otra parte, Cabero \& Barroso especifican que las tecnologías se han convertido en una importante herramienta didáctica la cual, "suscita la colaboración entre los alumnos, mejorando su motivación e interés por actividades educativas" (2015, p.
122). Hecho que reafirma que "el conocimiento básico sobre tecnología es imprescindible para entender y tener éxito en el mundo actual" (Siemens, 2016, p. 34).

Por lo tanto, si lo que se pretende es lograr un avance significativo hacia una sociedad de conocimiento, es necesario lograr un conglomerado de entes dedicados a esta área. Las experiencias exitosas según lo explica Camarena, se constituyen como "elementos que pueden converger en buenas prácticas" (2012, p. 34). En este orden de ideas, el propósito central de este artículo surge de las necesidades didácticas $y$ pedagógicas que se han detectado y que presentan los docentes al momento de impartir los lineamientos teórico-prácticos, y conceptos disciplinares del área de economía.

\section{Metodología}

En la estructura metodológica, el estudio se fundamentó desde un enfoque cualitativo de corte documental, con un tipo descriptivo; para la recolección de la información, se utilizó como instrumento una ficha de registro bibliográfico, en donde la técnica de análisis documental, se revisaron, recopilaron y analizaron 6 experiencias exitosas relacionadas a la enseñanza de finanzas y ciencias económicas, tomando como población a estudiantes universitarios en el contexto internacional y nacional. En relación directa con este planteamiento, Castillo explica que: El análisis documental es una operación
intelectual que da lugar a un subproducto o
documento secundario que actúa como
intermediario o instrumento de búsqueda
obligado entre el documento original y el
usuario que solicita información. El
calificativo de intelectual se debe a que el
documentalista debe realizar un proceso de
interpretación y análisis de la información de
los documentos y luego sintetizarlo (2005, p.
12)

El procedimiento llevado a cabo, permitió caracterizar e identificar diversos estudios que se han desarrollado en cuanto a 
la enseñanza aprendizaje de fundamentos teórico prácticos de finanzas internacionales en los últimos 21 años, dejando entrever el desarrollo en materia educativo de acuerdo con la incorporación y uso de tecnologías que se ha venido consolidando, en esta secuencia argumental, a continuación en la Tabla 1 se pueden apreciar las experiencias exitosas recopiladas (ver Tabla 2) y tomadas como fuente de análisis documental, conforme con la estructura y objetivos del estudio y revisión bibliográfica realizada previamente.

\section{Tabla 1}

Experiencias Exitosas en el Contexto Internacional

\begin{tabular}{|c|c|c|c|}
\hline Título de estudio & Autor & Ciudad/País & Año \\
\hline 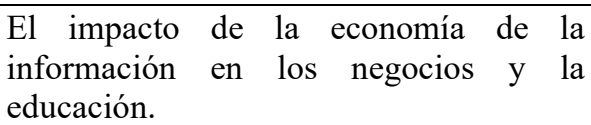 & (Mercado, 2010) & $\begin{array}{ll}\text { San } & \text { salvador, } \\
\text { Salvador } & \end{array}$ & 2010 \\
\hline $\begin{array}{l}\text { Las TIC en la enseñanza de las Ciencias } \\
\text { Económicas. }\end{array}$ & (Arónica, 2014) & Córdoba, Argentina & 2014 \\
\hline $\begin{array}{l}\text { El uso de las TIC en la enseñanza de la } \\
\text { Economía. }\end{array}$ & (Mur, 2015) & Madrid, España & 2015 \\
\hline
\end{tabular}

Nota. En la Tabla 1, se presentan 3 estudios a nivel internacional en el ámbito financiero en los últimos 15 años, elaboración propia (2021).

\section{Tabla 2}

Experiencias Exitosas en el Contexto Nacional

\begin{tabular}{llll}
\hline Título de estudio & Autor & Ciudad/País & Año \\
\hline $\begin{array}{l}\text { Las TIC y la educación en Colombia } \\
\text { durante la década del noventa: alianzas y } \\
\text { reacomodaciones entre el campo de las } \\
\text { políticas educativas, el campo académico }\end{array}$ & & $\begin{array}{l}\text { Antioquia, } \\
\text { Colombia }\end{array}$ & 2012 \\
y el campo empresarial & & & \\
Las TIC en la normativa para los (Mesa y & Forero, & Bogotá, Colombia & 2016 \\
programas de educación superior en 2016) & & & \\
$\begin{array}{l}\text { Colombia } \\
\text { Inclusión de las Tecnologí-as de la (Benjumea et al., }\end{array}$ & Bogotá, Colombia & 2017 \\
$\begin{array}{l}\text { Información y la Comunicación (TIC) en 2017) } \\
\text { el sistema financiero colombiano }\end{array}$ & & & \\
\hline
\end{tabular}

Nota. En la Tabla 2, se presentan 3 estudios a nivel nacional en el ámbito financiero en los últimos 10 años, elaboración propia (2021).

Para la realización de los datos se utilizó la técnica de revisión documental, de este modo los estudios recopilados, permitieron analizar el impacto de las Tecnologías en los procesos de educativos (ver Tabla 3), propios de las ciencias económicas y financieras, dejando entrever factores que evidencian ventajas y desventajas, al momento de integrar componentes digitales para el desarrollo de prácticas pedagógicas orientadas al mejoramiento de las competencias en materia económica, en los estudiantes universitarios desde sus primeros años de educación superior.
Los estudios recopilados se obtuvieron de diferentes fuentes y sitios especializados en divulgación de documentos académicos y científicos, revistas electrónicas de educación, así como en repositorios de universidades, teniendo en cuenta criterios, como que fueran estudios realizados del año 2000 en adelante, y fueran documentos como tesis de investigación y artículos académicos. De manera similar Castillo, recomienda recopilar y analizar información que se encuentre en multitud de formatos, "unos más 
accesibles, más reconocidos y mejor valorados académicamente que otros, entre estos: libros, revistas, actas de congresos, reportes técnicos, normas, tesis e Internet" $(2005$, p. 45). Este procedimiento es fundamental y punto de partida de cualquier investigación bibliográfica, ya que proporcionan una base y una visión más profunda del tema que se espere abordar.

Llevado al ámbito educativo y en estrecha relación con lo planteado por Cortés (2015) es preciso resaltar que el uso de las Tecnologías de la Información y
Comunicación se han establecido actualmente, como herramientas esenciales facilitadoras en los procesos de aprendizaje, dadas las múltiples ventajas y funcionalidades que estas prestan al desarrollo cognitivo, el aprovechamiento de recursos educativos y el efectivo apropiamiento de conocimientos; consecuente a ello, la integración y el uso de TIC permiten la optimización $y$ automatización de los datos obtenidos y entregados en los procesos académicos, el del desarrollo de proyectos administrativos y la consecución de sus objetivos.

Tabla 3

Fuentes de Búsqueda de la Información

\begin{tabular}{|c|c|c|c|}
\hline $\begin{array}{l}\text { Tipo de } \\
\text { documento }\end{array}$ & Obtenido de & Fuente & Año \\
\hline $\begin{array}{l}\text { Investigación, } \\
\text { tesis de } \\
\text { maestría }\end{array}$ & $\begin{array}{l}\text { Repositorio Institucional } \\
\text { de la Universidad del } \\
\text { salvador }\end{array}$ & http://ri.ues.edu.sv/id/eprint/12386/ & 2009 \\
\hline $\begin{array}{l}\text { Objeto de } \\
\text { conferencia } \\
\text { Simposio } \\
\text { Argentino de } \\
\text { Tecnología y } \\
\text { Sociedad } \\
\text { (STS) }\end{array}$ & $\begin{array}{l}\text { Repositorio Institucional } \\
\text { de la Universidad de la } \\
\text { Plata }\end{array}$ & http://sedici.unlp.edu.ar/handle/10915/41781 & 2014 \\
\hline $\begin{array}{l}\text { Artículo } \\
\text { revisión }\end{array}$ & $\begin{array}{l}\text { Fundación Dialnet } \\
\text { Universidad de la Rioja }\end{array}$ & $\begin{array}{l}\text { https://dialnet.unirioja.es/servlet/articulo? } \operatorname{codigo}=4 \\
690458\end{array}$ & 2015 \\
\hline Artículo & $\begin{array}{l}\text { Revista educación y } \\
\text { pedagogía. } \\
\text { Universidad de Antioquia }\end{array}$ & $\begin{array}{l}\text { https://revistas.udea.edu.co/index.php/revistaeyp/art } \\
\text { icle/view/14202 }\end{array}$ & 2012 \\
\hline $\begin{array}{l}\text { Artículo } \\
\text { revisión }\end{array}$ & $\begin{array}{l}\text { Revista de investigación } \\
\text { y pedagogía. Praxis \& } \\
\text { saber }\end{array}$ & $\begin{array}{l}\text { https://revistas.uptc.edu.co/index.php/praxis_saber/ } \\
\text { article/view/5219 }\end{array}$ & 2016 \\
\hline Artículo & $\begin{array}{l}\text { Revista UNIMAR } \\
\text { Universidad Mariana }\end{array}$ & $\begin{array}{l}\text { http://editorial.umariana.edu.co/revistas/index.php/ } \\
\text { unimar/article/view/1544 }\end{array}$ & 2017 \\
\hline
\end{tabular}

Nota. En la tabla 3 se presentan las diferentes fuentes de información, especificando el tipo de documento, la fuente y el año, elaboración propia (2021).

Cabe destacar que el aporte pedagógico subyacente del proceso realizado radica en poder analizar experiencias pedagógicas exitosas con el uso de TIC, ya que tal y como lo menciona Odetti, el análisis de experiencias exitosas es una "práctica que tiende a generar nuevas formas de gestión del conocimiento, poniendo de manifiesto que las transformaciones más importantes no son producto de las TIC, sino de las transformaciones en las formas de hacer las cosas que esas tecnologías posibilitan" (2015, p. 16).

\section{Resultados}

Para dar cumplimiento al objetivo central del estudio, el cual se orientó a determinar la influencia que han tenido las tecnologías educativas, en los procesos de enseñanza de finanzas internacionales, se procedió a realizar un proceso de análisis y revisión documental de seis estudios o 
experiencias exitosas en el ámbito educativo, las cuales tienen como característica principal el uso de tecnología, toda la información recopilada y analizada se buscó en fuentes y revistas especializadas en educación y tecnología, así como en repositorios de universidades tanto a nivel internacional como nacional.

Entre los principales resultados, se evidenció como en el contexto internacional se han venido desarrollando diversos estudios, que reflejan el impacto que ha venido teniendo las TIC en la enseñanza de las ciencias económicas, en fundamentos específicos como lo son las finanzas internacionales, este planteamiento se relaciona con lo planteado por Arónica la cual menciona que, a la hora de incorporar TIC es necesario tener en cuenta que, "no solo se debe enseñar las cuestiones referidas a la materia en sí (contenidos referidos a las ciencias económicas), si no también crear capacidades y habilidades para el manejo de esa información utilizando la herramienta elegida" (2014, p. 91).

De acuerdo con las recientes y múltiples modificaciones que ha sufrido el sistema educacional implantado y utilizado actualmente a nivel nacional por el MEN (2016) en las diferentes instituciones educativas, es de vital importancia, educar a los estudiantes conforme con los cambios y utilidades de las herramientas, como lo son las nuevas tecnologías de la información y la comunicación, las cuales han tenido una adherencia más que sobresaliente en los procesos académicos y educativos; permitiéndole a los docentes de diferentes áreas de estudio estar a la vanguardia del conocimiento, logrando generar nuevas y mejores experiencias de aprendizaje centradas en las necesidades pedagógicas, cognitivas y actitudinales de los estudiantes, como parte importante de su formación integral.

Por lo tanto, y posterior a la revisión documental de acuerdo con las experiencias exitosas con el uso de TIC, se puede afirmar que existe falencias pedagógicas en los docentes de Economía, al momento de realizar sus prácticas de enseñanza, se pudo constatar que hacen poco uso de componentes digitales, siendo necesario tal y como lo menciona Davis (1999) fomentar y profundizar en la integración de componentes digitales, para contribuir al mejoramiento y transformación de los proceso de enseñanza aprendizaje de las ciencias económicas.

Las TIC aportan un gran beneficio para crear nuevos ambientes educativos. Sin embargo, para ser efectivas deben estar entrelazadas con métodos tradicionales esto quiere decir que, los ambientes educativos deben ser promovidos por la integración y estimulación del desarrollo de ciertas habilidades intelectuales tales como el razonamiento, la resolución de problemas, la creatividad y la capacidad de aprender a aprender. Por lo tanto, este hecho fundamentalmente implica un uso armónico y funcional para un propósito del aprender específico en un dominio o una disciplina curricular.

\section{Finanzas Internacionales en un Mundo Globalizado}

En materia económica, y desde los planteamientos teóricos de Kozikowski "la economía de un país y su interrelación con el mundo es la base de estudio fundamental de las finanzas, porque involucra de por sí, a todo lo relacionado con el sistema financiero, las inversiones, el sistema cambiario entre otros" (2013, p. 56). Actualmente, el estudio de finanzas internacionales ha presentado un crecimiento exponencial, por lo que se constituye como un fundamento de vital importancia en la formación universitaria, ya que con estos lineamientos teóricos, los estudiantes podrán comprender el entorno internacional, y su interacción conforme la creciente globalización de la economía. Consistentemente con lo previamente expuesto, cabe destacar que Kozikowski, complementa su argumento explicando como: 


\begin{abstract}
El conocimiento de las finanzas internacionales permite a los tomadores de decisiones entender cómo los acontecimientos internacionales pueden afectar a su empresa y qué medidas deben poner en práctica para evitar los riesgos y aprovechar las oportunidades que ofrece la constante transformación del entorno mundial. La formación en el campo internacional ayuda a los ejecutivos y empresarios a anticipar los acontecimientos y tomar decisiones en forma proactiva $(2013$, p. 5$)$.
\end{abstract}

En este sentido, resulta importante mencionar en este punto, que los ejes temáticos tratados (ver Tabla 4), y de mayor relevancia en el estudio de finanzas internacionales con estudiantes universitarios, se pueden categorizar en dos grandes grupos, la economía internacional y las finanzas corporativas internacionales:

\section{Tabla 4}

Ejes de Estudio de Finanzas Internacionales

\begin{tabular}{|c|c|}
\hline $\begin{array}{c}\text { Economía } \\
\text { Internacional }\end{array}$ & \begin{tabular}{l}
\multicolumn{1}{c}{ Finanzas } \\
Corporativas \\
Internacionales
\end{tabular} \\
\hline 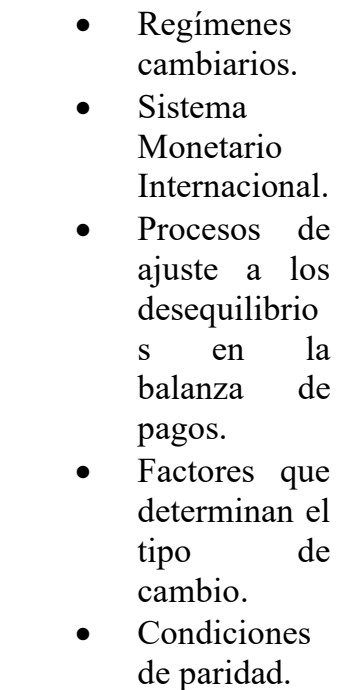 & $\begin{array}{ll}\text { - } & \text { Mercados } \\
\text { financieros } \\
\text { internacionales } \\
\text { - } \\
\text { Operaciones en } \\
\text { escala mundial. } \\
\text { Evaluación y } \\
\text { administración } \\
\text { del riesgo } \\
\text { cambiario. } \\
\text { - Financiamiento } \\
\text { internacional. } \\
\text { - Inversión en } \\
\text { portafolios } \\
\text { internacionales } \\
.\end{array}$ \\
\hline
\end{tabular}

Nota. En la tabla 4 se condensan los principales temas que se deben estudiar, tanto en economía como en finanzas corporativas internacionales desde la visión del autor. Tomado y adaptado de Kozikowski (2013).

Por lo tanto, teniendo en cuenta los contenidos temáticos contenidos en la tabla 4 , es de suma importancia que el estudiante universitario de economía, tal y como lo indica Glänzel \& Schubert (2019) pueda comprender el significado y funcionamiento de los sistemas económicos y financieros internacionales, ya que el dominio de esta materia es indispensable para su proyección de vida, como individuo que opera en una economía crecientemente integrada en la economía global y digital que se expande constantemente y permea todas las esferas en la cotidianidad de los seres humanos. Desde la postura teórica de Anaya la enseñanza y el aprendizaje de las finanzas es fundamental en primer lugar porque:

Determinan las formas y maneras en que se puede obtener recursos económicos una manera más fácil y eficiente, y segundo, la administración; ya que una vez que se han obtenido recursos, estos deben ser utilizados de forma eficiente para que produzcan y se conviertan a su vez en más recursos (2021, p. 345).

Según lo plantea Ontiveros, las TIC ha mantenido una relación estrecha con el sector financiero desde la aparición de los primeros sistemas de computación, a mediados del siglo XX. Diversos inventos tecnológicos han permitido dinamizar procesos específicos, como la gestión transaccional, la contratación en mercados o la interconexión con sistemas de pagos. "Desde la irrupción del mainframe a finales de los años 60, hasta las más recientes aplicaciones en la nube, las TIC han ido de la mano de la banca en su crecimiento" (2011, p. 22). Las TIC han tenido un impacto significativo en la educación, y más desde la declaración de pandemia emitida por la Organización Mundial de la salud, situación que llevó a los docentes a modificar sus métodos de enseñanza, por lo que vieron la necesidad de apoyarse en herramientas digitales para dar continuidad a sus prácticas pedagógicas.

\section{Las Tecnologías y su Relación con el Ámbito Educativo}

Ahora bien, resulta esencial analizar la relación existente entre el uso de la tecnología y el ámbito educativo, de acuerdo con la enseñanza y aprendizaje de fundamentos financieros y económicos. En relación directa con esto, Siemens (2016) desde su postura 
conectivista, explica como el aprendizaje (definido como conocimiento aplicable) puede residir fuera de otros (dentro de una organización o en una base de datos), el cual está enfocado en conectar conjuntos de información especializada, con las conexiones que le permiten adquirir mayor conocimiento a un determinado individuo.

En relación directa con los antes mencionado, Sierra-Llorente et al., hacen un análisis en donde mencionan que el aprendizaje en cuanto al uso, manejo y aplicación de la tecnología en los ambientes educativos resulta de vital importancia, ya que debido a los permanentes cambios en los currículos educativos, se exige y es necesaria una formación integral, permanente $\mathrm{y}$ continua acorde a los cambios en la dinámicas sociales actuales, "la educación siempre ha sido importante para el desarrollo, pero ha adquirido mayor relevancia en el mundo de hoy, teniendo en cuenta las profundas transformaciones en materia digital de acuerdo con los nuevos métodos virtuales educativos" (2016, p. 25).

Al respecto, Benjumea et al. (2017), explican que el estado colombiano se ha caracterizado por manejar unas políticas públicas coherentes en materia de tecnología educativa, si bien luego de que en el año 2000 se estableciera una estrategia de largo plazo orientada a lograr una penetración masiva de las TIC en Colombia mediante el documento CONPES 3072, se crearon las bases políticas del proyecto Agenda de Conectividad, el estado se ha visto en la tarea de fomentar vías políticas que no sólo mejoren las condiciones de vida de millones de colombianos, sino que también permitan "el acceso del país a importantes organismos internacionales como la OCDE" (Mesa \& Forero 2016). No obstante, aunque en el país se direccionen grandes proyectos en materia de infraestructura tecnológica, aún hay serias falencias en la forma como estas iniciativas pueden transversalizar las TIC en educación superior.

Integrar TIC en los procesos educativos es ahora un requisito que la sociedad le exige a todo sistema educativo, y son consideradas como herramientas facilitadoras de la gestión pedagógica, capaces de fomentar creatividad, e innovación, permitiendo en gran medida transformar las aulas, en ambientes educativos favorecen la didáctica y la lúdica para el goce de las diferentes actividades.

Atendiendo a este planteamiento, resulta relevante mencionar lo planteado por la Organización para la cooperación y el Desarrollo Económico (OCDE) al referir que las nuevas tecnologías abren la puerta a innumerables actividades de aprendizaje, disponibles en cualquier momento y lugar para todos, "fomentando el desarrollo de las capacidades del siglo XXI, facilitando el despliegue de prácticas de aprendizaje innovadoras y personalizar el aprendizaje para incluir a estudiantes que se hallan en riesgo de quedar rezagados" (OCDE, 2020, p. $8)$.

Por consiguiente, la incorporación de las TIC en la práctica pedagógica no solo se convierte en un recurso positivo para el estudiante, sino también para el docente, para lo cual es necesario que sus métodos pedagógicos sean herramientas básicas para la producción de conocimientos, en este sentido, la educación tiene la responsabilidad de estar al día con los avances tecnológicos y todo lo que estos, puedan aportar a la escuela, para poder formar ciudadanos integrales, competentes y comprometidos con el desarrollo social, económico y cultural de la sociedad actual.

\section{Conclusiones}

Las conclusiones subyacentes del proceso de revisión documental evidencian que, en el ámbito colombiano, existen pocos estudios investigativos, que busquen profundizar sobre el uso y funcionalidad de la TIC para la enseñanza de áreas como las Finanzas internacionales, dejando entrever, y abriendo el debate sobre la pertinencia de las prácticas y métodos pedagógicos que utilizan los docentes de economía al momento de 
desarrollar e impartir los contenidos teóricoprácticos en el aula.

Por otra parte, se puede concluir que los procesos de análisis documental resultan fundamentales para conocer el estado actual de la educación, permitiendo detectar ventajas y desventajas, que a la postre, le permitirán al docente mejorar sus métodos de enseñanza, este planteamiento se relaciona con lo expuesto por Parra, al mencionar que la importancia de la realización de procesos de revisión y análisis documental radica en que en conocer de su existencia, se posesiona de ella" (2012, p. 56).

Dentro de este ámbito, el análisis documental llevado a cabo permite concluir que la educación ha sido influenciada en gran manera por los efectos de las nuevas tecnologías y a su vez, ha sabido aprovecharlos respecto a la inclusión no solamente en las prácticas educativas o para el desarrollo de estrategias más interesantes y efectivas frente a los estudiantes, sino también, en la gestión administrativa de acuerdo con la calidad educativa de las instituciones educativas de educación superior.

Por consiguiente, la incorporación de las TIC según lo plantea Lion (2019) en la práctica pedagógica no solo se convierte en un recurso positivo para el estudiante sino también para el docente, siendo pertinente concluir que las tecnologías desde su apertura han mostrado tener un valor redundante de todas las actividades humanas, gracias a su factor innovador y optimizador dentro de las prácticas para el desarrollo humano.

En efecto, es válido afirmar que el uso de estrategias pedagógicas mediadas por TIC fomenta, el desarrollo intelectual y cognitivo en los estudiantes, hecho que es fundamental para que puedan generar nuevos aprendizajes, ya que al realizar actividades pedagógicas con contenidos didácticos e interactivos, diseñadas pensando en sus características, edad mental y grado de escolaridad, se pueden construir nuevos métodos de enseñanza, creando experiencias interactivas de aprendizaje, en donde docentes-estudiantes establecen una relación de mutua reciprocidad, construyendo en el proceso aprendizajes significativos de mayor calidad.

La innovación dentro del aula de clases es muy importante en la medida que los estudiantes perciben que las herramientas tecnológicas pueden aprovecharse dentro del proceso de aprendizaje para apoyarlo y fortalecerlo, en este sentido resulta importante estimular la implementación continua de este tipo de herramientas tecnológicas, hecho que sería un elemento clave en la forma en como el estudiante concibe la educación bajo estos métodos mediados con TIC en espacios y ambientes virtuales de aprendizaje.

Las TIC, han tenido un impacto significativo en el ámbito educativo, y más desde la declaración de pandemia emitida por la OMS (2020). Sin embargo, en la enseñanza de finanzas internacionales, existe poca cantidad de experiencias exitosas que reflejen el impacto de las tecnologías, hecho que evidencia falencias pedagógicas en los docentes de economía, siendo indispensable fomentar el uso de tecnologías y recursos digitales en sus prácticas pedagógicas y de enseñanza.

En conclusión, se pueden destacar los esfuerzos que se han venido desarrollando en el contexto colombiano, sin embargo existen pocos estudios e investigaciones que se orienten al uso de la tecnología para la enseñanza de finanzas internacionales, lo cual refleja la necesidad de incorporar componentes TIC en la planeación pedagógica de enseñanza de los docentes de economía en las instituciones educativas de educación básica y superior del país.

\section{Referencias}

Anaya, R. (2021). Los 6 elementos clave del aprendizaje de las finanzas. https://www.gestiopolis.com/6elementos-clave-aprendizaje-de-las-finanzas/

Arónica, S. F. (2014). Las TIC en la enseñanza de las Ciencias Económicas. Análisis enfocado a los profesionales en Ciencias Económicas que son docentes en las escuelas del nivel medio de Córdoba. Argentina. Universidad Nacional de Córdoba. http://sedici.unlp.edu.ar/bitstream/handle/10915/417 81/Documento_completo.pdf?sequence=1\&isAllow $\mathrm{ed}=\mathrm{y}$ 
Barragán, J., \& Vela, H. (2015). Impacto de las TICs en la Gestión de Negocios Internacionales. International Journal of Good Conscience., 3. http://www.spentamexico.org/v10-n3/A3.10(3)1833.pdf

Benjumea, M., Benjumea, M., \& Torres, C. (2017). Inclusión de las Tecnologías de la Información y la Comunicación (TIC) en el sistema financiero colombiano. Revista UNIMAR, 2, 255-267. http://editorial.umariana.edu.co/revistas/index.php/u nimar/article/view/1544/2314

Cabero, J., \& Barroso, J. (2015). Nuevos retos en tecnología educativa. Editorial Sintesís S.A. https://es.scribd.com/document/387819229/Technic al-Blog-Dia-Diagram-Mac-OSX-Yosemite-Fix

Camarena, P. (2012). Experiencias institucionales exitosas en educación a distancia. Universidad de Guadalajara, http://www.udgvirtual.udg.mx/apertura/index.php/a pertura/article/view/317/283

Castillo, L. (2005). Análisis documental. Universidad de Valencia. https://www.uv.es/macas/T5.pdf

Cortés, D. (2015). Experiencias significativas con uso de tic 2015. Experiencias significativas con uso de tic 2015 , $0(0)$ http://aprende.colombiaaprende.edu.co/es/expeTIC

Davis, N. (1999). Teacher education and information technology: challenges for teacher education. Journal of Information Techology for Teacher Education Scopus, 8(1). https://www.tandfonline.com/doi/pdf/10.1080/1475 9399900200052

Glänzel, W., \& Schubert, A. (2019). Trazando un camino entre lo simple y lo falso y lo complejo e inutilizable: Revisión de Henk F. Moed, Informetría evaluativa aplicada en la serie Análisis cualitativo y cuantitativo de la comunicación científica y académica. Springer Link, 19, 549-560. https://doi.org/10.1007/s11192$019-03045-8$

Kozikowski, Z. (2013). Finanzas Internacionales. Instituto Tecnológico y de Estudios Superiores de Monterrey Campus Toluca, https://www.academia.edu/35687810/Finanzas Inte rnacionales_por_Kozikowski_3ra_Edicion

Lion, C. (2019). Los desafíos y oportunidades de incluir tecnologías en las prácticas educativas. Análisis de casos inspiradores. Instituto Internacional de Planeamiento de La Educación, 4-42. https://www.buenosaires.iiep.unesco.org/sites/defaul $\mathrm{t} /$ files/archivos/analisis_comparativos__carina_lion_05_09_2019.pdf

López, D. (2006). El conocimiento y la comunicación: dos pilares fundamentales de la organización de la sociedad de la información. Scopus, 9(2). http://dspace.uhemisferios.edu.ec:8080/xmlui/bitstre am/handle/123456789/455/1244-5033-1-
PB.pdf?sequence $=1 \&$ is Allowed $=\mathrm{y}$

MEN. (2016). La innovación Educativa en Colombia. https://aprende.colombiaaprende.edu.co/ckfinder/us erfiles/files/Libro Innovacion MEN - V2.pdf

Mercado, J. (2010). El impacto de la economía de la información en los negocios y la educación. Repositorio Institucional de La Universidad Del Salvador. http://ri.ues.edu.sv/id/eprint/12386/

Mesa, F., \& Forero, A. (2016). Las TIC en la normativa para los programas de educación superior en Colombia. Universidad Pedagógica y Tecnológica de Colombia, $\quad 7(14)$. https://www.redalyc.org/jatsRepo/4772/4772499270 04/477249927004.pdf

Mur, F. (2015). El uso de las TICs en la enseñanza de la Economía. Universidad Complutense de Madrid. file://C:/Users/psdoc/Downloads/Dialnet-

EIUsoDeLasTICsEnLaEnsenanzaDeLaEconomia4690458 (2).pdf

Odetti, V. (2015). Experiencias valiosas con uso de TIC en las escuelas públicas de la Provincia de Buenos. Programa de Educación CIPPEC. https://www.cippec.org/wpcontent/uploads/2017/03/1194.pdf

Ontiveros, E. (2011). Las TIC y el sector financiero del futuro.

https://www.afi.es/webAfi/descargas/1410874/1448 777/las-tic-y-el-sector-financiero-del-futuro.pdf

Parra, C. Á. (2012). Las TIC y la educación en Colombia durante la década del noventa: alianzas y reacomodaciones entre el campo de las políticas educativas, el campo académico y el campo empresarial. Universidad de Antioquia Colombia, 24(62).

https://es.scribd.com/document/405609749/DialnetLasTICYLaEducacionEnColombiaDuranteLaDecad aDelNove-4161109

Sandoval, C. H. (2020). La Educación en Tiempo del Covid19 Herramientas TIC: El Nuevo Rol Docente en el Fortalecimiento del Proceso Enseñanza Aprendizaje de las Prácticas Educativa Innovadoras. Revista Tecnológica-Educativa Docentes 2.0,9(2), 24-31. https://doi.org/10.37843/rted.v9i2.138

Siemens, G. (2016). Conectivismo: Teoría del aprendizaje para la era Digital. http://ayudasydemascosas.blogspot.com/2016/08/co nectivismo-teoria-del-aprendizaje.html

Sierra-Llorente, J., Bueno-Giraldo, I., \& Monroy-Toro, S. (2016). Análisis del uso de las tecnologías TIC por parte de los docentes de las Instituciones educativas de la ciudad de Riohacha. Omnia, 22, 1-10 